\title{
Burst Astrophysics
}

\author{
Vladimir S. Netchitailo \\ Biolase Inc., Irvine, CA, USA \\ Email: v.netchitailo@sbcglobal.net
}

How to cite this paper: Netchitailo, V.S. (2017) Burst Astrophysics. Journal of High Energy Physics, Gravitation and Cosmology, 3, 157-166.

https://doi.org/10.4236/jhepgc.2017.32016

Received: December 19, 2016

Accepted: February 5, 2017

Published: February 8, 2017

Copyright $\odot 2017$ by author and Scientific Research Publishing Inc. This work is licensed under the Creative Commons Attribution International License (CC BY 4.0).

http://creativecommons.org/licenses/by/4.0/ (c) (i)

Open Access

\begin{abstract}
This article proposes an explanation for Fast Radio Bursts (FRBs) and Gamma Ray Bursts (GRBs) through the frames of Hypersphere World-Universe Model (WUM). WUM predicts that the concentration of protons and electrons in Intergalactic Plasma decreases inversely proportional to time and in present epoch equals to $0.25480 \mathrm{~m}^{-3}$. The energy density of Intergalactic Plasma relative to the critical energy density equals to $\Omega_{p} \approx 0.048$. Time delay of FRBs is calculated through these characteristics. A number of experimental results, including the redshift for FRB 150418, remarkable brightness for FRB 150807, and transient gamma-ray counterpart for FRB 131104 are explained. The distance to FRB 150807 object is predicted to be $\sim 800 \mathrm{Mpc}$. WUM holds that all macroobjects (galaxies, stars, and planets) contain a core composed of Dark Matter Particles. GRBs are explained as a sum of contributions of multicomponent dark matter annihilation. The spectra of such bursts depend on the composition of the Cores.
\end{abstract}

\section{Keywords}

Hypersphere World-Universe Model, Medium of the World, Intergalactic Plasma, Macroobjects Structure, Dark Matter Particles, Gamma-Ray Bursts, Fast Radio Bursts, FRB Time Delay

\section{Introduction}

Fast Radio Bursts (FRBs) are millisecond-duration radio signals originating from distant galaxies that have been discovered in recent years. These signals are dispersed in the Medium of the World. Together with redshift measurements, this dispersion can be used for fundamental physical investigations of Intergalactic Plasma.

There exists a close parallel between FRBs and Gamma Ray Bursts (GRBs). Both manifest themselves as mysterious flashes of energy that were quite challenging to study due to their short durations. Once the technology has advanced 
to allow rapid follow-up observations, both were found to have afterglows. The characteristics of the afterglows suggest that FRBs and GRBs may have something in common; furthermore, they may indeed be different flavors of the same event.

In Section 2 we present a short summary of experimental results and existent theoretical models in the field of Burst Astrophysics partially adapted from Wikipedia. In Section 3 we propose a new physical approach to FRBs and GRBs based on Hypersphere World-Universe Model (WUM). In Section 4 we calculate FRB time delay based on the predicted parameters of Intergalactic Plasma.

\section{Burst Astrophysics. Short Summary}

Wikipedia has this to say about Burst Astrophysics:

Gamma-ray bursts (GRBs) are extremely energetic explosions that have been observed in distant galaxies. They are the brightest electromagnetic events known to occur in the World [1]. Bursts can last from ten milliseconds to several hours [2] [3]. GRB 111209A is the longest lasting gamma-ray burst (GRB) detected by the Swift Gamma-Ray Burst Mission on December 9, 2011. Its duration is longer than 7 hours [2].

After an initial flash of gamma rays, a longer-lived "afterglow" is usually emitted at longer wavelengths ( $X$-ray, ultraviolet, optical, infrared, microwave and radio) [4]. GRBs were first detected in 1967. Following their discovery, hundreds of theoretical models were proposed to explain these bursts. Little information was available to verify these models until the 1997 detection of the first $X$-ray and optical afterglows and direct measurement of their redshifts. The true nature of these objects remains unknown, although the leading hypothesis is that they originate from the mergers of binary neutron stars or a neutron star with a black hole [5].

The means by which gamma-ray bursts convert energy into radiation remains poorly understood [6]. Any successful model of GRB emission must explain the physical process for generating gamma-ray emission that matches the observed diversity of light curves, spectra, and other characteristics [7]. Particularly challenging is the need to explain the very high efficiencies that are inferred from some explosions: some gamma-ray bursts may convert as much as half (or more) of the explosion energy into gamma-rays [8]. Early observations of the bright optical counterparts to GRB 990123 and to GRB 080319B [9] [10], have suggested that inverse Compton may be the dominant process in some events. In this model, pre-existing low-energy photons are scattered by relativistic electrons within the explosion, augmenting their energy by a large factor and transforming them into gamma-rays [11]. There is no theory that has successfully described the spectrum of all gamma-ray bursts [Gamma-ray burst].

Fast Radio Burst (FRB) is a high-energy astrophysical phenomenon manifested as a transient radio pulse lasting only a few milliseconds. These are bright, unresolved, broadband, millisecond flashes found in parts of the sky outside the Milky Way. The component frequencies of each burst are delayed by different amounts of time depending on the wavelength. This delay is described by a value 
referred to as a Dispersion Measure (DM) which is the total column density of free electrons between the observer and the source of FRB. Fast radio bursts have $D M s$ which are: much larger than expected for a source inside the Milky Way [12]; and consistent with propagation through ionized plasma [13].

The first FRB found was FRB 010621. The Lorimer Burst (FRB 010724) was discovered in archived data taken in 2001 by the Parkes radio dish in Australia. The fact that no further bursts were seen in 90 hours of additional observations implies that it was a singular event such as a supernova or merger of relativistic objects [13]. On 19 January 2015, astronomers from Parkes observatory reported that FRB 140514 had been observed for the first time live [14].

In 2007, just after the publication of the e-print with the first discovery, it was proposed that fast radio bursts could be related to hyperflares of magnetars [15]. In 2015 three studies supported the magnetar hypothesis [12] [16] [17]. In 2014 it was suggested that following dark matter-induced collapse of pulsars [18], the resulting expulsion of the pulsar magnetospheres could be the source of fast radio bursts [19].

On 18 April 2015, FRB 150418 was detected by the Parkes observatory and within hours, several telescopes including the Australia Telescope Compact Array caught an "afterglow" of the flash, which took six days to fade [20]. The Subaru telescope was used to find what was thought to be the host galaxy and determine its redshift and the implied distance to the burst [21]. However, the origin of the burst was soon disputed by P. K. G. Williams and E. Berger who claim that the emission instead originates from an active galactic nucleus that is powered by a supermassive black hole with dual jets blasting outward from the black hole [22]. It was also noted that what was thought to be an "afterglow", never goes away, meaning that it cannot be associated with the fast radio burst [23] [Fast radio burst]. We will discuss FRB 150418 in Section 4.

On August 2015, FRB 150807 of remarkable brightness was detected by the Parkes observatory. Astronomers report on a mildly dispersed (DM $266.5 \pm 0.1$ $\left.\mathrm{pc} \mathrm{cm}^{-3}\right)$, exceptionally intense $(120 \pm 30 \mathrm{Jy})$, linearly polarized, scintillating burst that was directly localized to $9 \operatorname{arcmin}^{2}$. The burst scintillation suggests weak turbulence in the ionized intergalactic medium. The localization of FRB 150807 can be used to estimate the distance at which it was emitted, if it can associated with a star or a galaxy [24]. We will discuss FRB 150807 in Section 4.

The most intriguing result was obtained by J. J. DeLaunay, et al. [25]. They report the discovery of a transient gamma-ray counterpart to the fast radio burst FRB 131104, the first such counterpart to any FRB. The transient counterpart has duration $T_{90} \gtrsim 100 \mathrm{~s}$ and fluence $S_{\gamma} \approx 4 \times 10^{-6} \operatorname{ergcm}^{-2}(15-150 \mathrm{keV})$, increasing the energy budget for this event by more than a billion times; at the nominal $z \approx 0.55$ redshift implied by its dispersion measure, the burst's gammaray energy output is $E_{\gamma} \approx 5 \times 10^{51} \mathrm{erg}$. We will discuss this astronomical event in Section 3.

The discovery that some FRBs are accompanied by energetic gamma-ray transients dramatically alters the basic picture of these events. They have modest energy in radio flash ( $E_{\text {radio }} \sim 4 \times 10^{41}$ erg in case of FRB 131104) in comparison 
with gamma-ray energy that is more than $10^{9}$ times greater, with dramatic implications for source models and a substantial improvement in the prospects for long-lived counterparts, including X-ray and radio afterglows [25].

\section{Hypersphere World-Universe Model}

Hypersphere World-Universe Model (WUM) discusses the possibility of all Macroobject cores to be composed of Dark Matter Particles (DMP) with predicted masses of $1.3 \mathrm{TeV}, 9.6 \mathrm{GeV}, 70 \mathrm{MeV}, 340 \mathrm{keV}$, and $3.7 \mathrm{keV}$. The energy density of all macroobjects in the World $\Omega_{M O}$ relative to the critical energy density is $\Omega_{M O} \approx 0.024$ [26].

One of the most important DMP for galaxies is spin-0 boson which we dubbed ELOP that is preon dipole with mass $340 \mathrm{keV}$ [27]. Dissociated ELOPs can only exist at nuclear density or at high temperatures. ELOP breaks into two preons with mass about $m_{p r}=\frac{1}{3} m_{e} \cong 170 \mathrm{keV} / \mathrm{c}^{2}$ and charges $e_{p r}=\frac{1}{3} e$ which we took to match the Quark Model ( $m_{e}$ and $e$ are mass and charge of electrons). In particle physics, preons are postulated to be "point-like" particles, conceived to be subcomponents of quarks and leptons [28]. ELOPs are analogous to electron-positron pairs with charge $\frac{1}{3} e$.

In addition to ELOP discussed above, we offer another type of DMPspin-0 boson which we dubbed DIRAC that is in fact magnetic dipole with mass $70 \mathrm{MeV}$ [27]. Dissociated DIRACs can only exist at nuclear densities or at high temperatures. A DIRAC breaks into two Dirac monopoles with mass $\frac{m_{e}}{2 \alpha} \cong 35 \mathrm{MeV} / \mathrm{c}^{2}$ and charge $\mu=\frac{e}{2 \alpha}$ ( $\alpha$ is fine-structure constant).

In WUM we derive scaling solutions for a free and an interacting Fermi gas. The numerical values for maximum energy of the galaxies' shell made up of preons and monopoles in the present epoch are: $E_{p r} \approx 5.3 \times 10^{54} \mathrm{~J}$ and $E_{\text {mon }} \approx 1.3 \times 10^{50} \mathrm{~J}$ respectively [27].

According to WUM cores and shells of all macroobjects are growing in time until they reach the critical stability, at which point they detonate. The energy released during detonation is produced by annihilation of DMP. The detonation process does not destroy the macroobject; it's rather analogous to Solar flares.

In frames of WUM the experimental results for Gamma-Ray Bursts are explained thusly:

- The nature of these objects - cores and shells of galaxies made up from DMP;

- The means by which bursts convert energy into radiation-the annihilation of DMP;

- The very high efficiencies that are inferred from some explosions;

- The burst's gamma-ray energy output $E_{\gamma} \approx 5 \times 10^{44} \mathrm{~J}$ [25] that is 10 orders of magnitude smaller than the maximum energy of preons' shell $E_{p r} \approx 5.3 \times 10^{54} \mathrm{~J}$ [27];

- The spectrum of all gamma-ray bursts can be explained by the composition 
of cores and shells made up from DMP;

- A longer-lived "afterglow" that is usually emitted at longer wavelengths (Xray, ultraviolet, optical, infrared, microwave and radio) is a result of longlived processes developing in the cores and shells after detonation.

The duration of ultra-long gamma-ray burst 111209A is longer than 7 hours, implying this event has a different kind of progenitor than normal long GRBs. According to the authors of paper [2]: The host galaxy of GRB 111209A has not been resolved by the Hubble Space Telescope: only the GRB afterglow was visible, and GRB $111209 A$ traces the location of a putative (very) metal poor galaxy at large distance $(z=0.677)$. At this distance, this galaxy would not have been detected without the GRB which occurred in it.

A. J. Levan, et al. have this to say about ultra-long duration gamma-ray bursts: The long durations may naturally be explained by the engine driven explosions of stars of much larger radii than normally considered for GRB progenitors which are thought to have compact Wolf-Rayet progenitor stars [29]. It was first proposed that the progenitor of this event was a blue supergiant star with low metallicity [2].

In frames of WUM, this event can be explained by the galaxies' shell made up of monopoles:

- The burst's gamma-ray isotropic energy for GRB $111209 \mathrm{~A} \quad E_{\text {iso }} \approx 5.8 \times 10^{46} \mathrm{~J}$

[2] is about 2200 times less than the maximum energy of monopoles' shell $E_{\text {mon }} \approx 1.3 \times 10^{50} \mathrm{~J}$ [27]. This scenario is favored because of the necessity to supply enough mass to the central engine over duration of thousands of seconds [2];

- Gamma rays with energy in the range $20 \mathrm{keV}<E<1400 \mathrm{keV} \mathrm{[3]} \mathrm{are} \mathrm{a} \mathrm{con-}$ sequence of monopoles and preons' annihilation.

The described picture is consistent with experimental results for Fast Radio Bursts:

- The observations that sources of FRB are old galaxies;

- FRBs are the result of preons' plasma instability triggering shock waves of gigantic electrical currents and generating a huge amount of energy in transient radio pulses lasting only a few milliseconds;

- All other DMP can start annihilation process as the result of preons' shell instability and give rise to the gamma-radiation with different emission lines in spectra of galaxies.

- Gamma rays with energy less than $170 \mathrm{keV}$ are a consequence of preons' annihilation.

In our opinion, the annihilation of DMP is the most probable process that can generate huge amounts of energy in a very short time. The described galaxies bursts are analogous to the solar bursts which are bright emissions of photons with energies in excess of $100 \mathrm{MeV}$ [30].

\section{Fast Radio Bursts}

One of the most important parts of the Medium is Intergalactic Plasma with the 
concentration of protons and electrons that is decreasing inversely proportional to time. It has the energy density $\Omega_{p}$ relative to the critical energy density $\Omega_{p} \approx 0.048$ in the present epoch. In this section we calculate a time delay of FRB based on these characteristics of the Intergalactic Plasma.

In our model, protons and electrons have identical concentrations in the $\mathrm{Me}$ dium of the World [26]:

$$
n_{p}=n_{e}=\frac{2 \pi^{2}}{a^{3}} \frac{m_{e}}{m_{p}} \times Q^{-1}=0.25480 \mathrm{~m}^{-3}
$$

where $a=2 \pi a_{0}, a_{0}$ is the classical electron radius and $Q$ is a dimensionless time-varying fundamental parameter which equals to: $Q=0.759972 \times 10^{40}$ in present epoch [26].

A. Mirizzi, et al. found that the mean diffuse intergalactic plasma density is bounded by $n_{e} \lesssim 0.27 \mathrm{~m}^{-3}$ [31] corresponding to the WMAP measurement of the baryon density [32]. The mediums' plasma density (4.1) is in good agreement with the estimated value [31].

Low density intergalactic plasma has plasma frequency $v_{p l}$ [26]:

$$
v_{p l}=\frac{c}{a}\left(\frac{m_{e}}{m_{p}}\right)^{1 / 2} \times Q^{-1 / 2}=4.5322 \mathrm{~Hz}
$$

where $c$ is the electrodynamic constant in Maxwell's equations. Photons with energy smaller than $E=h v_{p l}$ cannot propagate in plasma; thus $h v_{p l}$ is the smallest amount of energy a photon may possess. This amount of energy can be viewed as a particle (we will name it phion), whose frequency-independent effective "rest energy" $E_{p h i}$ equals to [26]:

$$
E_{p h i}=E_{0}\left(\frac{m_{e}}{m_{p}}\right)^{1 / 2} \times Q^{-1 / 2}=1.8743 \times 10^{-14} \mathrm{eV}
$$

where $E_{0}$ is the fundamental unit of energy: $E_{0}=h c / a$ and $h$ is Planck constant. In WUM, a photon is a constituent phion with rest energy $E_{p h i}=h v_{p l}$ and total energy $E=h v\left(v \gg v_{p l}\right)$.

According to WUM, phions are fully characterized by their four-momentum $\left(\frac{E}{C}, \boldsymbol{p}\right)$ that satisfies the following equation:

$$
\left(\frac{E}{c}\right)^{2}-\boldsymbol{p}^{2}=\operatorname{In} v=\left(p_{p h i}\right)^{2}
$$

where the invariant is, in fact, the gravitoelectrostatic charge $p_{p h i}$ squared, and $E$ is the gravitoelectromagnetic charge [26]. Phions are moving in the Medium of the World with a group velocity $v_{g r}$ which can be found from (4.4):

$$
\frac{v_{g r}^{2}}{c^{2}}=1-\frac{E_{p h i}^{2}}{E^{2}} \text {. }
$$

Consider a photon with initial frequency $v_{\text {emit }}$ and energy $E_{\text {emit }}$ emitted at time $\tau_{\text {emit }}$ when the radius of the hypersphere world in the fourth spatial di- 
mension was $R_{\text {emit }}$. The photon is continuously losing kinetic energy as it moves from galaxy to the Earth until time $\tau_{\text {obsv }}$ when the radius is $R_{\text {obsv }}=R_{0}$. The observer will measure $v_{\text {obsv }}$ and energy $E_{\text {obsv }}$ and calculate a redshift:

$$
1+z=\frac{v_{\text {emit }}}{v_{\text {obsv }}}=\frac{E_{\text {emit }}}{E_{\text {obsv }}} \text {. }
$$

Recall that $\tau_{\text {emit }}$ and $\tau_{\text {obsv }}$ are cosmological times (Ages of the World at the moments of emitting and observing), both measured from the Beginning of the World. $\tau_{\text {obsv }}$ equals to the present age of the world. A light travel time-distance to a galaxy $d_{L L T}$ equals to

$$
d_{L T T}=c\left(\tau_{\text {obsv }}-\tau_{\text {emit }}\right)=c t_{L T T}=R_{0}-R_{\text {emit }} .
$$

Let's calculate photons' traveling time $t_{p h}$ from a galaxy to the Earth taking into account that $E_{p h i} \ll E$ :

$$
t_{p h}=\frac{1}{c} \int_{R_{\mathrm{emit}}}^{R_{0}} \frac{\mathrm{d} r}{\sqrt{1-\frac{E_{p h i}^{2}}{E^{2}}}}=t_{L T T}+\Delta t_{p h}
$$

where $\Delta t_{p h}$ is photons' time delay relative to the light travel time $t_{L L T}$ that equals to:

$$
\Delta t_{p h}=\frac{1}{2 c} \int_{R_{\text {emit }}}^{R_{0}} \frac{E_{p h i}^{2}}{E^{2}} \mathrm{~d} r .
$$

All observed FRBs have redshifts $z<1$. It means that we can use the Hubble's law: $d_{L T T}=R_{0} z$. Then

$$
R_{\text {emit }}=(1-z) R_{0} .
$$

Phions' energy squared at radius $R$ between $R_{\text {emit }}$ and $R_{0}$ equals to (4.3):

$$
E_{p h i}^{2}=\frac{m_{e}}{m_{p}} \frac{a}{R} E_{0}^{2} .
$$

According to WUM, photons' energy on the way from galaxy to an observer can be expressed by the following equation:

$$
E=z E_{\text {obsv }}+(1-z) \frac{R_{0}}{R} E_{\text {obsv }}=z \frac{R_{0}}{R} E_{\text {obsv }}\left(\frac{1-z}{z}+\frac{R}{R_{0}}\right)
$$

which reduces to $E_{\text {emit }}$ at (4.10) and to $E_{\text {obsv }}$ at $R=R_{0}$. Placing the values of the parameters (4.10), (4.11), (4.12) into (4.9), we have for photons' time delay:

$$
\begin{aligned}
\Delta t_{p h}= & \frac{1}{2 z^{2}} \frac{c}{a} \frac{m_{e}}{m_{p}} \frac{1}{v^{2}} \int_{1-z}^{1} \frac{x \mathrm{~d} x}{\left(x+\frac{1-z}{z}\right)^{2}}=\frac{1}{2 z^{2}} \frac{c}{a} \frac{m_{e}}{m_{p}} \frac{1}{v^{2}} \int_{\frac{1-z^{2}}{z}}^{\frac{1}{z}} \frac{\left(y-\frac{1-z}{z}\right) \mathrm{d} y}{y^{2}} \\
= & \frac{1}{2 z^{2}}\left[\ln \left(\frac{1}{1-z^{2}}\right)-\frac{z^{2}}{1+z}\right] \frac{c}{a} \frac{m_{e}}{m_{p}} \times \frac{1}{v^{2}}=\frac{4.61}{z^{2}}\left[\ln \left(\frac{1}{1-z^{2}}\right)-\frac{z^{2}}{1+z}\right] \times\left(\frac{v}{1 \mathrm{GHz}}\right)^{-2} \\
& \text { where } x=R / R_{0} \text { and } y=x+\frac{1-z}{z} . \text { Taking } z=0.492 \text { [33] we get the calculated }
\end{aligned}
$$


value of photons' time delay

$$
\Delta t_{p h}^{c a l}=2.189 \times\left(\frac{v}{1 \mathrm{GHz}}\right)^{-2}
$$

which is in a good agreement with experimentally measured value [33]

$$
\Delta t_{p h}^{\mathrm{exp}}=2.438 \times\left(\frac{v}{1 \mathrm{GHz}}\right)^{-2} .
$$

The difference between these values is $10.2 \%$. It is worth to note that in our calculations there is no need in a dispersion measure (DM) which is the total column density of free electrons between the observer and the source of FRB.

It is important to note that according to WUM the relative energy density of the Intergalactic plasma is $4.8 \%$ that is in a very good agreement with experimentally found value $4.9 \% \pm 1.3 \%$ [33]. The developed analysis based on WUM is consistent with all experimental results obtained by authors of [33].

The line-of-sight free electron column density for FRB 150807, measured in units of DM, is $266.5 \pm 0.1 \mathrm{pc} \cdot \mathrm{cm}^{-3}$. This substantially exceeds the expected foreground Milky Way DM, predicted to be $70 \pm 20 \mathrm{pc} \cdot \mathrm{cm}^{-3}$ along the burst sightline. According to the authors of paper [24]:

"The localization of FRB 150807 can be used to estimate the distance at which it was emitted, if we can associate the FRB with a star or a galaxy. The deepest archival images of the sky localization area contain nine objects brighter than a Ks-band magnitude of 19.2 (11): three stars and six galaxies. The brightest galaxy is at a distance between 1 and 2 Gpc estimated from its photometric redshift. The other galaxies are factors of $>6$ fainter than the brightest. Through a comparison of their infrared magnitudes with empirical and theoretical distributions of galaxy luminosities at different distances, they are all expected to be $>500 \mathrm{Mpc}$ distant".

In our opinion, based on the equation (4.13) and measured value DM, they should look for an old galaxy (not a star) which has the redshift $z=0.19 \pm 0.02$ and the distance about $800 \mathrm{Mpc}$. Hopefully the performed calculations will help astronomers to find the right source of FRB 150807.

Very recently, 16 additional bright bursts in the direction of FRB 121102 were detected (see [34] and references inhere). According to the authors of paper [34]: This repeating FRB is inconsistent with all of the catastrophic event models put forward previously for hypothetically non-repeating FRBs. Here, we propose a different model, in which highly magnetized pulsars travel through the asteroid belts of other stars.

In frames of WUM, these repeating FRBs can be explained by the galaxy flares analogous to Solar flares as it is described in Section 3.

Transient Astrophysics is a rapidly growing field, now operating across all wavelengths, observed from the ground and in space. Using multi-wavelength observations allows us to study the various components of the World in extraordinary detail. With the high sensitivity and wide-field coverage of the Square Kilometre Array, large samples of explosive transients are expected to be discov- 
ered [35]. Hypersphere World-Universe Model can serve as a basis for Transient Astrophysics.

\section{Acknowledgements}

I thank the anonymous referees for useful comments and suggestions that have led to an overall improvement of the manuscript. Special thanks to my son Ilya Netchitailo who helped shape it to its present form.

\section{References}

[1] NASA (2014) Gamma Rays. http://missionscience.nasa.gov/ems/12_gammarays.html

[2] Gendre, B., et al. (2013) The Ultra-Long Gamma-Ray Burst 111209A: The Collapse of a Blue Supergiant? The Astrophysical Journal, 766, 30. https://doi.org/10.1088/0004-637X/766/1/30

[3] Stratta, G., et al. (2013) The Ultra-Long GRB 111209A-II. Prompt to after Glow and After Glow Properties. arXiv:1306.1699.

[4] Vedrenne, G. and Atteia, J.-L. (2009) Gamma-Ray Bursts: The Brightest Explosions in the Universe. Springer, New York. https://doi.org/10.1007/978-3-540-39088-6

[5] Nakar, E. (2007) Short-Hard Gamma-Ray Bursts. Physics Reports, 442, 166. https://doi.org/10.1016/j.physrep.2007.02.005

[6] Stern, B.E. and Poutanen, J. (2004) Gamma-Ray Bursts from Synchrotron SelfCompton Emission. Monthly Notices of the Royal Astronomical Society, 352, L35. https://doi.org/10.1111/j.1365-2966.2004.08163.x

[7] Fishman, G.J. (1995) Gamma-Ray Bursts: An Overview. http://apod.nasa.gov/diamond_jubilee/papers/fishman.html

[8] Fan, Y. and Piran, T. (2006) Gamma-Ray Burst Efficiency and Possible Physical Processes Shaping the Early Afterglow. Monthly Notices of the Royal Astronomical Society, 369, 197. https://doi.org/10.1111/j.1365-2966.2006.10280.x

[9] Racusin, J.L., et al. (2008) Broadband Observations of the Naked-Eye Gamma-Ray Burst GRB 080319B. Nature, 455, 183. https://doi.org/10.1038/nature07270

[10] Liang E.P., et al. (1999) GRB 990123: The Case for Saturated Comptonization. The Astrophysical Journal, 519, L21. https://doi.org/10.1086/312100

[11] Wozniak, P.R., et al. (2009) Gamma-Ray Burst at the Extreme: The Naked-Eye Burst GRB. 080319B. Astrophysical Journal, 691, 495. https://doi.org/10.1088/0004-637X/691/1/495

[12] Masui, K., et al. (2015) Dense Magnetized Plasma Associated with a Fast Radio Burst. Nature. 528,523. https://doi.org/10.1038/nature15769

[13] Lorimer, D.R., et al. (2007) A Bright Millisecond Radio Burst of Extragalactic Origin. Science, 318, 777. https://doi.org/10.1126/science.1147532

[14] Sim, H. (2015) Cosmic Radio Burst Caught Red-Handed. Royal Astronomical Society, London.

https://www.ras.org.uk/news-and-press/2578-cosmic-radio-burst-caught-red-hande $\underline{\mathrm{d}}$

[15] Popov, S.B. and Postno, K.A. (2007) Hyperflares of SGRs as an Engine for Millisecond Extragalactic Radio Bursts. arXiv:0710.2006.

[16] Champion, D.J., et al. (2015) Five New Fast Radio Bursts from the HTRU High Latitude Survey: First Evidence for Two-Component Bursts. arXiv:1511.07746. 
[17] Kulkarni, S.R., Ofek, E.O. and Neill, J.D. (2015) The Arecibo Fast Radio Burst: Dense Circum-Burst Medium. arXiv:1511.09137.

[18] Bramante, J. and Linden, T. (2014) Detecting Dark Matter with Imploding Pulsars in the Galactic Center. Physical Review Letters, 113, Article ID: 191301. https://doi.org/10.1103/PhysRevLett.113.191301

[19] Fuller, J. and Ott, C. (2015) Dark Matter-Induced Collapse of Neutron Stars: A Possible Link Between Fast Radio Bursts and the Missing Pulsar Problem. Monthly Notices of the Royal Astronomical Society: Letters, 450, L71. https://doi.org/10.1093/mnrasl/slv049

[20] Keane, E.F., et al. (2016) The Host Galaxy of a Fast Radio Burst. Nature, 530, 453. https://doi.org/10.1038/nature17140

[21] Subaru Telescope (2016) New Fast Radio Burst Discovery Finds Missing Matter in the Universe. http://www.subarutelescope.org/Pressrelease/2016/02/24a/index.html

[22] Williams, P.K.G. and Berger, E. (2016) Cosmological Origin for FRB 150418? Not So Fast. http://newton.cx/ peter/wp/wp-content/uploads/2016/02/note-rev1.pdf

[23] Pulliam, C. (2016) Fast Radio Burst Afterglow Was Actually a Flickering Black Hole. Harvard-Smithsonian Center for Astrophysics, Cambridge. https://www.cfa.harvard.edu/news/2016-10

[24] Ravi, V., et al. (2016) The Magnetic Field and Turbulence of the Cosmic Web Measured Using a Brilliant Fast Radio Burst. https://doi.org/10.1126/science.aaf6807

[25] DeLaunay, J.J., et al. (2016) Discovery of a Transient Gamma-Ray Counterpart to FRB 131104. arXiv:1611.03139v1.

[26] Netchitailo, V.S. (2015) Hypersphere World-Universe Model. Journal of High Energy Physics, Gravitation and Cosmology, 2, 593. https://doi.org/10.4236/jhepgc.2016.24052

[27] Netchitailo, V.S. (2015) 5D World-Universe Model. Multi-Component Dark Matter. Journal of High Energy Physics, Gravitation and Cosmology, 1, 55. https://doi.org/10.4236/jhepgc.2015.12006

[28] D’Souza, I.A. and Kalman, C.S. (1992) Preons: Models of Leptons, Quarks and Gauge Bosons as Composite Objects. World Scientific, Singapore. https://doi.org/10.1142/1700

[29] Levan, A.J., et al. (2013) A New Population of Ultra-Long Duration Gamma-Ray Bursts. arXiv:1302.2352.

[30] Overview of Solar Flares (2008). http://hesperia.gsfc.nasa.gov/hessi/flares.htm

[31] Mirizzi, A., Raffelt, G.G. and Serpico, P.D. (2006) Photon-Axion Conversion in Intergalactic Magnetic Fields and Cosmological Consequences. arXiv:0607415.

[32] Spergel, D.N., et al. (2003) First Year Wilkinson Microwave Anisotropy Probe (WMAP) Observations: Determination of Cosmological Parameters. arXiv:0302209.

[33] Keane, E.F., et al. (2016) A Fast Radio Burst Host Galaxy. https://doi.org/10.1038/nature 17140

[34] Dai, Z.G., Wang J.S., Wu, X.F. and Huang, Y.F. (2016) Repeating Fast Radio Bursts from Highly Magnetized Pulsars Traveling through Asteroid Belts. https://doi.org/10.3847/0004-637x/829/1/27

[35] Fender, R., et al. (2015) Transient Astrophysics with the Square Kilometre Array. arXiv:1507.00729. 
Submit or recommend next manuscript to SCIRP and we will provide best service for you:

Accepting pre-submission inquiries through Email, Facebook, LinkedIn, Twitter, etc. A wide selection of journals (inclusive of 9 subjects, more than 200 journals)

Providing 24-hour high-quality service

User-friendly online submission system

Fair and swift peer-review system

Efficient typesetting and proofreading procedure

Display of the result of downloads and visits, as well as the number of cited articles Maximum dissemination of your research work

Submit your manuscript at: http://papersubmission.scirp.org/

Or contact jhepgc@scirp.org 